\title{
Pericardiectomía, en paciente con taponamiento cardiaco por Hemangiosarcoma
}

\author{
Karen Vega-Benavides $^{1} \bowtie$, Mauricio Jiménez-Soto ${ }^{1}$ \\ 1 Hospital Especies Menores y Silvestres, Escuela Medicina Veterinaria, Universidad Nacional. Email: \\ karenluvega@hotmail.com, drjimenezsoto@hotmail.com
}

Hemangiosarcoma, tumor maligno que se origina en las células endoteliales de vasos sanguíneos, son el bazo, la aurícula derecha, el hígado y el tejido subcutáneo las localizaciones más frecuentes; con alta tasa de metástasis. Los síntomas que produce dependen de la localización. Debilidad, intolerancia al ejercicio o colapso, puede deberse a sangrados o insuficiencia cardiaca por efusión en pericardio. En la mayoría de los casos, la citología del derrame pericárdico no es diagnóstica, al igual que citologías de la masa, es por esto que los diagnósticos definitivos suelen ser pos cirugía, o post mortem. Cuando el principal signo es causa del taponamiento, se recomienda pericardiocentesis de emergencia para estabilizar el paciente, y posteriormente un abordaje quirúrgico de pericardiectomía, en este caso subtotal; en dicho procedimiento se realiza el corte por debajo del nivel de los nervios frénicos, en ambos lados, lo que permite la liberación del miocardio del pericardio, que es poco expansible, y permite una diástole y sístole más eficientes. En casos de hemangiosarcomas cardiacos, estudios recientes indican que la combinación de manejo quirúrgico con quimioterapia ofrece el mejor pronóstico, incluso en presencia de metástasis. Canino, macho, geriátrico. Se presenta a consulta en choque hipovolémico, con diagnóstico previo de ascitis por hepatopatía. Se procede a estabilización del paciente con terapia de fluidos y oxígeno, así como monitorización de signos vitales, hematología y electrolitos; en la revisión de emergencia se realiza ecografía FAS abdominal, lo que evidencia efusión sero-sanguinolenta (por punción), además de múltiples nodulaciones en el hígado, el bazo y linfonos mesentéricos; y FAS torácico, al presentar taponamiento cardiaco, con presencia de masa en zona atrio- ventricular derecha (pared). Se realiza pericardiocentesis de emergencia, la cual permite estabilización del paciente en su volemia (presión media estable) y capacidad ventilatoria (saturación oxígeno). Se indica a los propietarios las opciones de abordaje médico y quirúrgico, además de los diagnósticos diferenciales, y el pronóstico del paciente, basado en hallazgos previos. Al presentar el paciente una mejora evidente posterior al manejo del taponamiento, se decide someterlo toracotomía izquierda y se realiza la pericardiectomía subtotal, se toma muestra por punción aguja fina de la masa (no fue diagnostica), y se coloca drenaje torácico, el cual se retira al tercer día pos quirúrgico y se da de alta al paciente. En los exámenes posteriores a la cirugía el animal presenta anemia leve (hematocrito 29 \%) y línea linfoide en límite inferior. Se explica opción de quimioterapia, con evidencia bibliográfica de mejorar sobrevida, pero no aceptan. Una vez cicatrizada la herida, y terminado el periodo de tratamiento antibiótico, antinflamatorio y analgésico, se inicia con Prednisolona (se retiró posteriormente por vómito). La calidad de vida del paciente mejoró, se mostraba activo, relatan los propietarios que su actividad y convivencia con otros perros y la familia volvió a ser como antes. Se realizaron chequeos de hematología, ecografía torácica y abdominal cada 15 días, después de ser dado de alta a la hospitalización, y después cada mes (según signos y evolución del paciente). Al quinto mes pos quirúrgico el paciente presenta anemia marcada (hematocrito $15 \%$ ); sin embrago, su condición general es buena, por lo que se realiza transfusión sanguínea, siendo claros en que se mejoraba un signo, pero la condición del paciente iba en detrimento; el paciente mejoró y nuevamente fue dado de alta. Al sexto mes

Autor para correspondencia Karen L. Vega-Benavides: karenluvega@hotmail.com 
pos quirúrgico se presenta nuevamente, a pesar de que sus parámetros y ecografía torácicos no se muestran con mayor alteración, se evidencia anemia (hematocrito $13 \%$ ), y aumento de tamaño en masas hepáticas, en este momento se decide eutanasia del paciente. Conclusiones: En presencia de tumores malignos, en este caso torácico, incluso relacionados a vasos grandes o corazón, a pesar de ser de pronóstico reservado a malo, no siempre es mandatorio realizar eutanasia inmediata; cuando se tiene propietarios comprometidos y con los que se puede mantener buena comunicación, se puede ofrecer manejos paliativos que suelen mejorar la calidad y expectativa de vida en los animales. La medicina veterinaria cada vez debe ser más especializada, y ofrecer opciones a nuestros pacientes. 